\title{
Special issue of PAAA devoted to CIARP 2019
}

\author{
Heydi Méndez-Vázquez ${ }^{2}$. José Ruiz-Shulcloper ${ }^{1}$
}

Accepted: 12 November 2020 / Published online: 26 November 2020

(c) Springer-Verlag London Ltd., part of Springer Nature 2020

This special issue is devoted to the 24th Iberoamerican Congress on Pattern Recognition (CIARP, Congreso Iberoamericano de Reconocimiento de Patrones). CIARP is the yearly international event of a series of conferences on Pattern Recognition organized since 1995 by the national associations for Pattern Recognition and related areas in Iberoamerican countries. The 2019 edition of CIARP has been held in Havana, Cuba, from October 28 to 31, 2019, and this special issue includes the extended versions of selected CIARP 2019 papers.

To this CIARP, 128 contributions from 23 countries were submitted. An international Program Committee consisting of 144 prestigious scientists from 17 countries had the task to rigorously evaluate the submissions. At the end of the reviewing process, 72 papers from 14 countries were accepted, with a $45.31 \%$ rejection rate.

The presentation type, oral or poster, was established by taking into account a number of factors, such as the wider or narrower size of the interested audience, but the level of the paper was not related to the type of presentation. In any case, all contributions were presented in plenary sessions. Besides the 72 accepted submissions, the scientific program of CIARP 2019 also included the contributions of five outstanding invited speakers, namely Ingela Nyström (Uppsala University, Sweden), Petra Perner (Institute of Computer Vision and Applied Computer Sciences IBaI Leipzig, Germany), Alberto Del Bimbo (Università degli Studi di Firenze, Italy), Massimo Tistarelli (Computer Vision Laboratory Università di Sassari, Italy), and Jean-François Bonastre (Université d'Avignon, France). The proceedings of the conference were published in Lectures Notes on Computing Sciences, "Progress in Pattern Recognition, Image Analysis, Computer Vision, and Applications", LNCS 11,896.

José Ruiz-Shulcloper

jshulcloper@uci.cu

1 Universidad de Las Ciencias Informáticas, Havana, Cuba

2 Advanced Technologies Application Center (CENATAV), Havana, Cuba
As usual in the CIARP process for elaborating special issues from the best papers presented at the conference, by taking in consideration the scope of the Pattern Analysis and Application Journal (PAAA) and the topics treated in the CIARP 2019 papers, the recommendations about the suitability of CIARP 2019 papers for the PAAA journal, done by the Program Committee members who handled reviewing of the conference papers, the average scores assigned to each contribution by its referees, and the quality of the presentations done by the authors at the conference, we selected 11 top CIARP 2019 contributions and invited their corresponding authors to submit extended versions of their CIARP 2019 papers to PAAA by June 2020. For the sake of clarity, we informed the authors that even if their papers were recommended for publication, their inclusion in the special issue could not be a priori guaranteed. In fact, all papers for which acceptance was recommended were reviewed by part of the previous reviewers of these papers and others of the Program Committee members. As a result of the reviewing process, 10 papers were accepted for publication in this special issue.

The first fours papers in the special issue are related to applications to Medicine:

In "Classification among Healthy, Mild Cognitive Impairment and Alzheimer's disease subjects based on Wavelet Entropy and relative Beta and Theta power", from Jorge Esteban Santos Toural, Arquímedes Montoya Pedrón, and Enrique Juan Marañón Reyes, is proposed a method for classifying Alzheimer's disease, mild cognitive impairment, and healthy subjects. The classification is made by means of a support vector machine with polynomial kernel and a two-layer neural network based on features obtained from wavelet decomposition. The obtained results show that the method could be the basis for the implementation of a diagnosis-support quantitative tool oriented to aid in clinical diagnosis.

In "Analysis of cancer in histological images: employment an approach based on genetic algorithm", Daniela Taino, Matheus Ribeiro, Guilherme Freire Roberto, Geraldo F.D. Zafalon, Marcelo Z. do Nascimento, Thaína Aparecida Tosta, Alessandro S. Martins, and Leandro A. Neves. 
propose an approach based on genetic algorithm to evaluate a significant number of features, selection methods, and classifiers in order to provide an acceptable association for the diagnosis and pattern recognition of non-Hodgkin lymphomas and colorectal cancer.

In "A Hermite polynomial algorithm for detection of the lesion in lymphoma images", Alessandro Martins, Leandro Neves, Paulo Faria, Leonardo Longo, Taína Tosta, and Marcelo do Nascimento investigated algorithms based on multiscale and multidimensional fractal geometry with colour models for classification of lymphoma images. The authors used Hermite polynomial classifier and machine learning algorithms with fractal features extracted from the colour models and separate channels obtained from histological images that show to be relevant for the binary and multiclass classification of the lesions in lymphoma images.

In "A convolutional oculomotor representation to model parkinsonian fixational patterns from magnified videos", Isail Salazar Acosta, Said David Pertuz Arroyo, William Omar Contreras López, and Fabio Martínez present a novel imaging biomarker for PD assessment that models ocular fixational movements, recorded with conventional cameras. The proposed imaging-based descriptor properly captures known disease tremor patterns, suggesting a successful PD characterisation from fixational eye motion patterns using ordinary videos.

The successive two papers deal with fingerprint:

In "Document scanners for minutiae-based palmprint recognition. A feasibility study", Manuel Aguado Martínez, José Hernández-Palancar, Katy Castillo-Rosado, Christof Kauba, Simon Kirchgasser, and Andreas Uhl study the feasibility of using document scanners as a cheaper option to acquire palmprints for minutiae-based matching systems. A new high-resolution palmprint dataset was established, and an enhancement algorithm was proposed to attenuate the negative effect of creases in the process of minutiae extraction.

In "An ensemble of fingerprint matching algorithms based on cylinder codes and mtriplets for latent fingerprint identification", Danilo Valdes-Ramirez, Miguel Angel Medina-Pérez, and Raúl Monroy propose a fusion of two lower-level fingerprint matching algorithms using a supervised classifier. The proposal achieves higher identification rates than each lower-level algorithm and that their fusion using traditional combining approaches.

There are three papers related to other biometric traits such as face, speech, and gait recognition:

In "Face Spoofing Detection via Ensemble of Classifiers towards Low-Power Devices", Rafael Vareto, Matheus Diniz, and William Robson Schwartz propose a low-computational cost method for face spoofing detection. The proposed method is one of the few methods associating features derived from both spatial and frequency image domains. Experiments on recent and well-known datasets under same and cross-database settings show that although the methodology is geared for resource-limited singleboard computers, it can produce significant results.

In "Multichannel Spectrograms for Speech Processing Applications Using Deep Learning Methods", Tomas Arias Vergara, Philipp Klumpp, Juan Camilo VasquezCorrea, Elmar Noeth, Juan Rafael Orozco Arroyave, and Maria Schuster propose a methodology to combine three different time-frequency representations of the signals into 3D-channel spectrograms to analyse speech in two different applications: (1) automatic detection of speech deficits in cochlear implant users and (2) phoneme class recognition to extract phone-attribute features.

In "Simple and Efficient Gait Recognition Method for Challenging Environments", from Vítor Lima and William Schwartz, a method for gait recognition based on the extraction of characteristics from the skeleton of the person is presented. Two variants of the approach are presented: one is based on features extracted from the body parts and the combination of two classifiers, and the other uses a multilayer perceptron to learn a representation based on the detected body parts in each image; temporal aggregation is performed by majority voting. Proposed approaches have been successfully tested on the CASIA $\mathrm{A}$ and $\mathrm{B}$ datasets.

Finally, there is another paper that proposes a method for action recognition:

In "Second Order Motion Descriptors for Efficient Action Recognition", Reinier Oves García, Eduardo Morales, and Enrique Sucar introduce a new set of second-order motion representations capable of capturing both: geometrical and kinematic properties of the motion. Besides, an strategy capable of reducing training times without sacrificing the performance when using the I3D two-stream CNN is presented. The experiments presented in UCF101 and HMDB51, two of the most challenging datasets for action recognition, show competitive results.

We take this opportunity to express our sincere gratitude to all the authors who submitted their works to the special issue and the Program Committee members who handled this second reviewing process that provided important comments, definitely useful for the authors to improve their contributions. We are also indebted to the Senior Editor, Dragos Calitoiu, and the Editor in Chief of this journal, Sameer Singh, who kindly accepted our proposal for the special issue and were always available to help us in answering questions done by contributors and solving problems before they could have created troubles during the reviewing process.

We hope that readers of PAAA will enjoy reading this special issue and will find it as a useful and interesting reference for their future work. 
Heydi Méndez-Vázquez and José Ruiz-Shulcloper. Guest Editors.
Publisher's Note Springer Nature remains neutral with regard to jurisdictional claims in published maps and institutional affiliations. 